\title{
Chemical Reactions on Metal-supported Hexagonal Boron Nitride Investigated with Density Functional Theory
}

\author{
Pauline Bacle, Ari Paavo Seitsonen, Marcella lannuzzi, and Jürg Hutter*
}

\begin{abstract}
Nanotechnology, in order to become ultimately efficient, requires achieving the construction of elemental structures at the atomistic precision. One way toward this goal is using templated surfaces as support for directed synthesis. The nanomesh, a single-atom thick layer of hexagonal boron nitride on $\mathrm{Rh}(111)$ [Corso et al., Science 2004, 303, 217], has appeared as one candidate that provides a periodically corrugated structure on the nanometre scale. We present density functional theory studies where we investigate various properties of the nanomesh, ranging from intrinsic defects to covalent functionalisation with hydroxyl radicals. Further we study selective dehalogenation of an organic molecule (I6-CHP) on the nanomesh. This molecule adsorbs at particular sites of the template and has been activated for $\mathrm{C}-\mathrm{C}$ coupling in recent experiments via dissociation of its halogen ligands. In all cases we find explicit templating effects, either in full agreement with experimental studies or predicting novel phenomena. The studies are evidence for the predictive power of modern electronic structure simulations and the insight that can be gained when used together with experiments on complex chemical structures.
\end{abstract}

Keywords: Density functional theory calculations · Functionalisation · Nanomesh · Surfaces · Templating

\section{Introduction}

Hexagonal boron nitride, $h$-BN, is isoelectronic to graphite and the two substances share many structural properties. Of special interest are the single layer i.e. atom-thin structures that can be built from both materials. This kind of templated layer is desirable in the field of nano-devices, where the precise positioning and the control of individual molecules are of importance. $h$-BN layers that build templating surfaces are a promising starting point to obtain defined arrangements on a large scale. Such layers have been reported to show variations in interaction strength on a nanometre scale and, due to the large band gap of $h$-BN, are able to provide an efficient electronic decoupling from the supporting metal surface. ${ }^{[1-4]}$

Monolayers of $h$-BN have been grown on transition metal surfaces by chemical vapour decomposition of small precursor molecules, e.g. borazine. ${ }^{[5]}$ On hot metallic surfaces a spontaneous formation of a single uniform epitaxial monolayer is observed. Recently, well-characterised
${ }^{\star}$ Correspondence: Prof. J. Hutter

Institut für Chemie

Universität Zürich

Winterthurerstrasse 190, CH-8057 Zürich

Tel.: +41446354491

E-mail Hutter@Chem.uzh.ch single-layer $h$-BN structures have been grown on several transition metals, such as $\mathrm{Ni}(111),{ }^{[6]} \mathrm{Cu}(111),{ }^{[6,7]} \mathrm{Ru}(0001),{ }^{[8]}$ $\operatorname{Rh}(111),{ }^{[5,9]} \operatorname{Pd}(111),{ }^{[10]} \operatorname{Ag}(111),{ }^{[11]}$ and $\operatorname{Pt}(111){ }^{[12]}$ Using similar recipes, also graphene structures on most Pt-group transition metal surfaces have been obtained. ${ }^{[8,13]}$ The resulting long-range superstructure depends on the mismatch in the unit cell length of the free-standing monolayer and the metal surface. In $h$-BN/ $\mathrm{Rh}(111)$ a lattice mismatch of $-7.0 \%$ leads to a strongly corrugated structure with a periodicity of $3.22 \mathrm{~nm}$, the so-called $n a$ nomesh ${ }^{[9]}$ The highly regular hexagonal arrangement corresponds to a coincidence lattice of $13 \times 13 h$-BN on $12 \times 12 \mathrm{Rh}$ unit cells. The nanomesh is characterised by 'pores' of about $2 \mathrm{~nm}$ diameter that strongly interact with the metal and elevated regions, where the interaction with the metal is weak, that form the connected 'wire' network. The corrugation pattern, shown in Fig. 1, corresponds to a modulation of the B-N bond length and, thereby of the electronic properties of the monolayer. Such a variation in bond lengths contributes to the spatial modulation of the electrostatic potential above the monolayer that is responsible for trapping molecules. ${ }^{[7,14-16]}$

The $h-\mathrm{BN} / \mathrm{Rh}(111)$ nanomesh is a very stable structure that withstands temperatures of $1000 \mathrm{~K}$ and can be exposed to liquids without losing its properties. Reversibility of the modification of the structure using atomic hydrogen has also been demonstrated. ${ }^{[17]}$ The spatial variation of the electronic structure can be used for directed functionalisation or to induce specific chemical reactions by exploiting the template provided by the nanomesh. The selective adsorption of molecules on the nanomesh

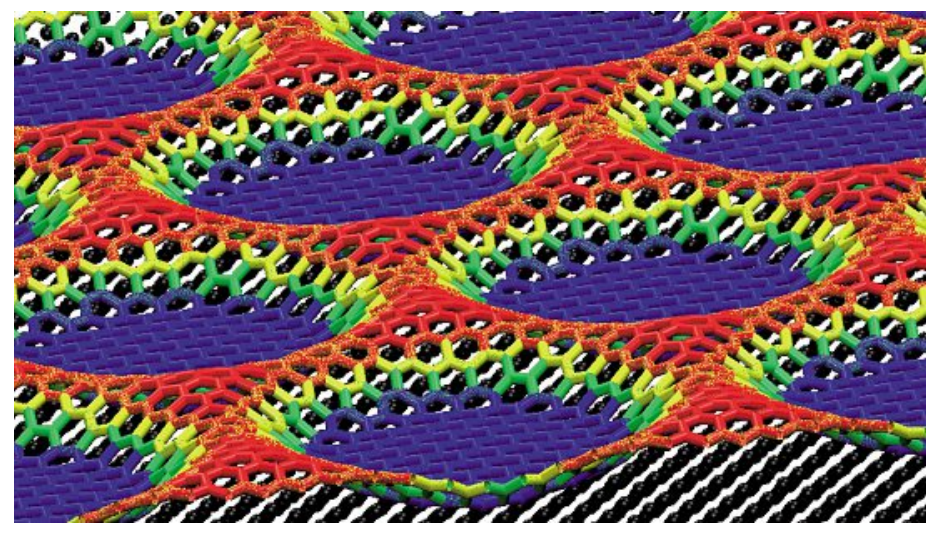

Fig. 1. Structure of the nanomesh of $h$-BN/ $\mathrm{Rh}(111)$. The colour code of the $h$-BN monolayer reflects the height of the $B$ and $\mathrm{N}$ atoms with respect to the substrate. The blue areas correspond to the pore, the red to the wire regions, and the atoms coloured with yellow and green form the so-called 'rim'. 
has been already studied for a few organic molecules ${ }^{[9,16,18]}$ and in great detail for water.[19-22]

In this work, we present three significant examples of the effects of corrugation and of mediated interaction with the metal on chemical processes leading to the functionalisation of the nanomesh. The first example is the adsorption of a highly symmetric molecule (hexa-iodo-cyclohexa-phenylene), which undergoes a specific pattern in dehalogenation that can only be understood in connection with the underlying template. The second example is related to the formation and aggregation of vacancy defects on the monolayer that can become active centres for further site selective processes. The third example is the activation of possible reaction paths for the dissociative coupling of water molecules to the nanomesh, where we demonstrate increased stabilisation of the products due to the presence of the metal.

\section{Computational Details}

All calculations were performed using Kohn-Sham density functional theory (DFT) within the Gaussian plane wave (GPW) formalism as implemented in the Quickstep module of the CP2K program package. ${ }^{[23,24]}$ Dual-space pseudopotentials[25-27] are used to describe the interaction of valence electrons with atomic cores. The pseudopotentials for boron and nitrogen assume three and five valence electrons, respectively. The atomic cores of $\mathrm{Rh}$ atoms are described by a large-core pseudopotential with nine valence electrons. Single- (for Rh), double- (B, N, $\mathrm{C}$ and $\mathrm{I}$ ) and triple- ( $\mathrm{H}$ and $\mathrm{O}$ ) zeta basis sets including polarised function are employed to describe the valence electrons. The plane wave (PW) energy cutoff for the expansion of the density is set to $500 \mathrm{Ry}$. The Brillouin zone is sampled only at the $\Gamma$ point. Exchange and correlation $(\mathrm{XC})$ terms are evaluated with the $\mathrm{PBE}$ or revised $\mathrm{PBE}^{[28-30]}$ generalised gradient approximation (GGA) XC functional. Long-range dispersion interactions are added using the DFT-D3 formalism. ${ }^{[31]}$ The XC functional and its derivative are calculated on the same uniform density grid that is used for the Hartree energy and is defined by the choice of the PW energy cutoff. In order to improve the accuracy in the evaluation of the XC terms a nearest neighbour smoothing procedure is used. More details about these techniques are illustrated in ref. [32]. The Fermi-Dirac smearing of occupation numbers with a $300 \mathrm{~K}$ electronic temperature is used in all calculations. Broyden density mixing [33] is used to facilitate smooth convergence within a reasonable number of iterations. Periodic boundary conditions are applied in all calculations. In the slab models, interactions with periodic images in the direction perpendicular to the exposed surface are avoided by adding about $20 \AA$ of vacuum space above the slab. The size of the unit cell used in the calculations of the full nanomesh is $32.1195 \AA$ in the lateral directions. This corresponds to a $12 \times 12$, four-layer rhodium slab with a cell parameter of $3.785 \AA$ and an adsorbed $13 \times 13 h$-BN monolayer.

To simulate the dissociative reaction of water on the nanomesh, instead, a $6 \times 6 \mathrm{Rh}(111)$ slab of seven layers is used as substrate. In these models, a one to one commensurate structure is obtained by stretching the $h$-BN overlayer to the $\mathrm{Rh}(111)$ lattice constant. The $h$-BN layer can be arranged with nitrogen on top of a $\mathrm{Rh}$ atom and $\mathrm{B}$ on the next hollow site, or with nitrogen occupying a hollow site. The registries with $\mathrm{N}$ atop are the most stable structures and serve as models for the pore environment, whereas when $\mathrm{N}$ occupies a hollow site, we have a model for the wire environment. A complete assessment of this computational setup can be found in ref. [34].

\section{Templating: Formation of Covalent Networks on the Nanomesh}

A possible path to building functionalised graphene-containing structures in a controlled way is the bottom-up synthesis from small molecules toward patterned assemblies. Thereby covalent carbon-carbon bonds are created between the molecular units. Two recent examples of such Ullmann-like coupling reactions on metal surfaces involve the dehalogenation of the initial molecules to form graphene nanoribbons ${ }^{[35]}$ and polymeric networks on the coinage metals. ${ }^{[36]}$

Motivated by recent experiments on the adsorption of CHP $\left(5,5^{\prime}, 5^{\prime \prime}, 5^{\prime \prime \prime}, 5^{\prime \prime \prime \prime}, 5^{\prime \prime \prime \prime}\right.$-hexaiodo-cyclohexa-m-phenylene, Fig. 2) molecules on the nanomesh, we have conducted DFT calculations in order to better understand the experimental observations. ${ }^{[37]}$ Here we discuss further aspects of the DFT results.

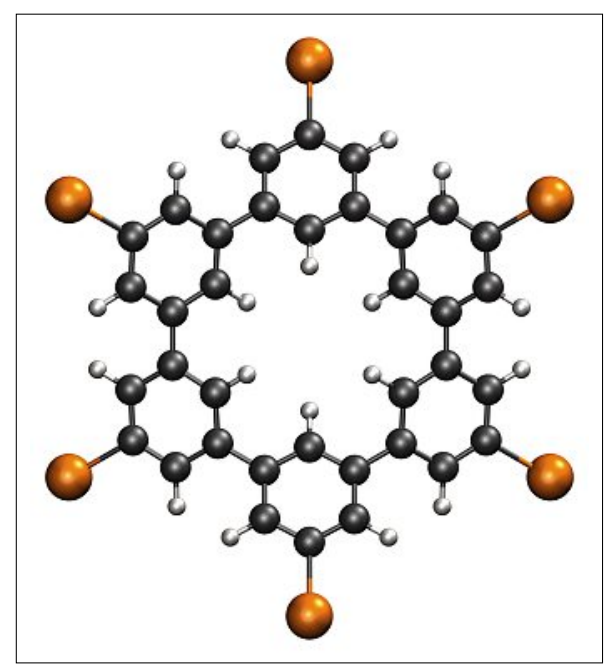

Fig. 2. The molecular structure of $5,5^{\prime}, 5^{\prime \prime}, 5^{\prime \prime \prime}, 5^{\prime \prime \prime \prime}, 5^{\prime \prime \prime \prime ' '-h e x a i o d o-c y c l o h e x a-~}$ $\mathrm{m}$-phenylene (16-CHP). The geometry is nonplanar, with alternating buckling of the iodo-phenylene groups.

We find that CHP adsorbs in the pore with a binding energy of $88 \mathrm{kcal} / \mathrm{mol}$. Considering the size of the molecule and that basically all the atoms are located almost planarly above the $h$-BN layer, the interaction is principally of van der Waals type. What is remarkable in the adsorption geometry is that three out of the six iodine atoms lie clearly higher, by $0.33,0.95$ and $1.03 \AA$ than the centre of the molecule, or the carbon atoms, and the three other atoms are lying close to the plane formed by the phenyl rings. This pattern is alternating between the raised and planar iodines. We denote the in-plane atoms as 'A' and the lifted ones as 'B' (Fig. 3).

The dissociation of an iodine atom from the molecule can be initiated either with a voltage pulse from the tip of a scanning tunnelling microscope or by heating the system. The dissociation can follow two different kind of pathways, depending on whether the iodine is detached from an A or B site. We start two separate calculations, one for dissociation from an A site and another one from $\mathrm{B}$. The former is obtained placing the dissociated iodine atom above a boron atom on the nanomesh in the pore, and upon the relaxation the iodine atom basically remained laterally at this site.

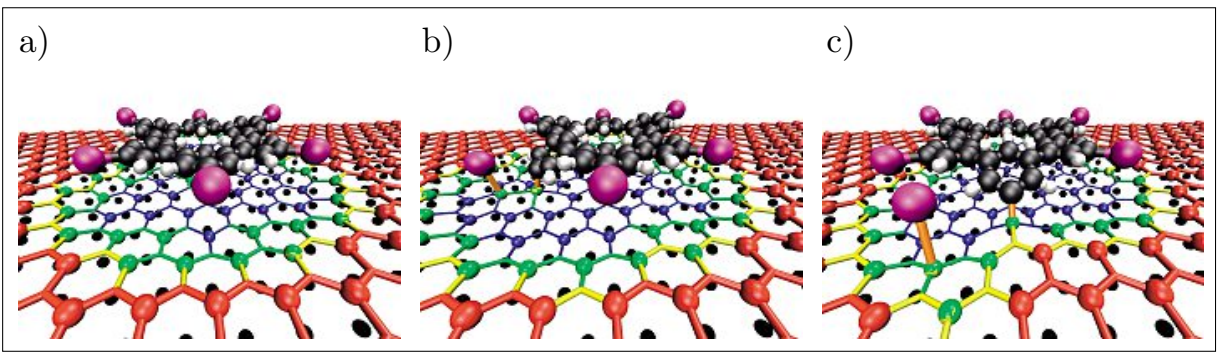

Fig. 3. a) Adsorbed CHP in the pore of the nanomesh. Dissociated structure after extraction of an iodine at the b) A and c) B site; see text for definitions. 
From this configuration, a nudged-elastic band (NEB) simulation is started where the final image is the dissociated structure in Fig. 3c. On the other hand, to simulate the same dissociation reaction on a B site, the dissociated iodine atom of the final configuration is positioned above a boron atom somewhat higher on the wire region of the nanomesh because there is not enough space in the pore. The iodine then relaxes laterally to a different position, returning relatively close to the rest of the molecule, but without reforming a chemical bond. The nanomesh also responds to the dissociation of iodine atoms by forming a bond with the rest of the molecule via a boron site. This boron atom is lifted from the rest of the pore, and the phenyl group, from which the iodine comes, bends strongly downwards.

During imaging in scanning tunnelling microscopy (STM) the limit of tip-induced dehalogenation is reached if the 5,5",5"'-I3-CHP is formed, i.e. a complete dissociation at B sites occurs. This is also the most often observed molecule when thermal dehalogenation is performed. The selectivity is surprising as there is no difference in stability calculated for single, isolated molecules for any order of dehalogenation. However, a series of calculations of CHP molecules on the nanomesh clearly establishes a preferred order of dehalogenations, leading to the final I3-CHP found in experiment. The key point is the anchoring of the I5-CHP after the first dehalogenation on a boron atom. Further strong interactions of radical type carbon atoms with boron atoms after the second and third step of multiple dehalogenations are then only possible in B sites.

We also investigate the energetics of the isolated iodine atoms. These are obtained upon the dissociation from the molecule and are present on the surface until two of them meet to form $\mathrm{I}_{2}$ and then desorb. The adsorption energy of one iodine in the pore is $31 \mathrm{kcal} / \mathrm{mol}$, and on the wire only $4 \mathrm{kcal} / \mathrm{mol}$. This again demonstrates the strong difference in reactivity between the pore and wire regions. The adsorption site is above a boron atom, which gets lifted somewhat from the local structure of the nanomesh.

\section{Formation of $\mathrm{B}$ and $\mathrm{N}$ Vacancies}

The exposure of hexagonal boron nitride single layers to low energy ions leads to the formation of vacancy defects that are mobile at elevated temperatures. Recently, intercalation processes of $\mathrm{Ar}$ and $\mathrm{Rb}$, forming the so-called 'nanotent' structures, have been reported after exposure of the $h$-BN nanomesh to low energy ions. ${ }^{[38,39]}$ The ion bombardment sputters out boron and nitro-

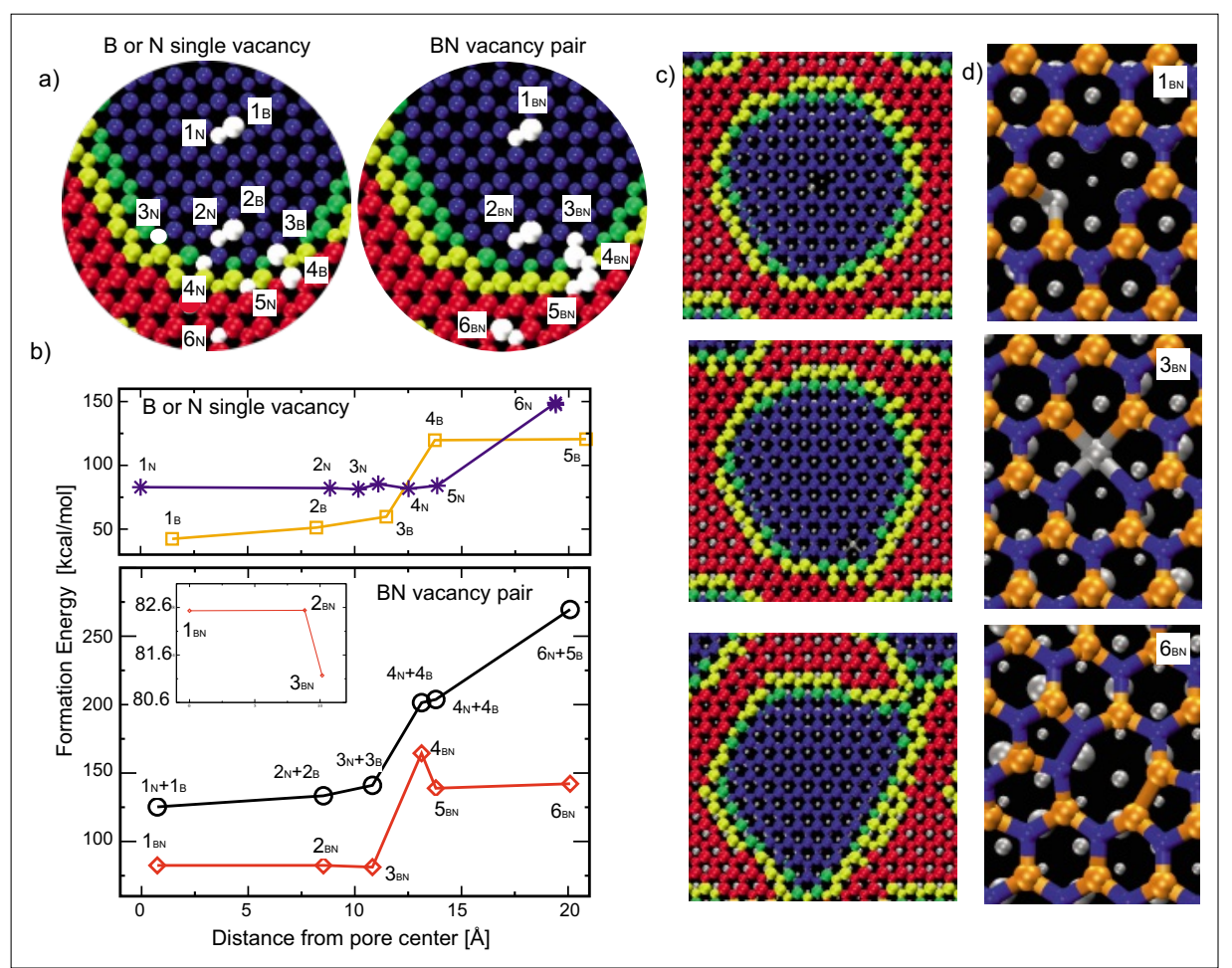

Fig. 4. a) Top view of a portion of the nanomesh unit cell where the B atoms (larger spheres) and $\mathrm{N}$ atoms (smaller spheres) are coloured according to their height above the Rh surface (same colour code as in Fig. 1). The labelled white spheres indicate the selected sites of the vacancies. b) Formation energy of vacancies as a function of the distance from the pore centre. The labels correspond to the sites indicated in panel a). In the bottom graph, in red the formation energies of the vacancy pairs, in black the sum of two single vacancy formation energies, computed individually. The inset shows a zoom-in on points 1, 2, and 3. c), d) Top view of three optimised structures with one vacancy pair. c) Global view of the nanomesh unit shell with same colour code as in Fig. 1. d) Ball-and-stick zoom-ins that show the local reconstruction around the defect ( $\mathrm{N}$ are drawn as blue, $\mathrm{B}$ as orange, and $\mathrm{Rh}$ as silver spheres).

gen atoms, leaving vacancy defects within the $h$-BN layer, which are visible at the rim and at the wire of the nanomesh by STM. Interestingly, an annealing of the defective system leads to self-healing processes, where the defects seem to gather, first forming larger defects and then inducing reconstruction of the monolayer (formation of holes and self-healing). Formation, stability and mobility of $\mathrm{B}$ and $\mathrm{N}$ vacancies on the nanomesh are of great interest in the view of employing the defective template as active substrate for further functionalisation processes.

We perform DFT calculations of single and vacancy pairs at different sites within the $h$-BN super-honeycomb structure. The results are summarised in Fig. 4. After removing one $\mathrm{B}$ or $\mathrm{N}$ atom, or one $\mathrm{BN}$ pair, we fully relax the structure and compare to the pristine nanomesh. The vacancy formation energy is obtained in terms of the chemical potentials of the atomic species, $\mu_{\mathrm{B}}$ and $\mu_{\mathrm{N}}$, as

$$
E_{\mathrm{f}}=E_{\mathrm{dnm}}-E_{\mathrm{nm}}+n_{\mathrm{vN}} \mu_{\mathrm{N}}+n_{\mathrm{vB}} \mu_{\mathrm{B}},
$$

where the number of removed $\mathrm{B}$ and $\mathrm{N}$ atoms per nanomesh unit cell is $n_{\mathrm{vB}}$ or $n_{v N}$, respectively, and $E_{\mathrm{nm}}$ and $E_{\mathrm{dnm}}$ are the total energies of pristine and defective nanomesh. The reference chemical potentials of $\mathrm{B}$ and $\mathrm{N}$ are determined under the constraint $\mu_{\mathrm{BN}}=\mu_{\mathrm{B}}+\mu_{\mathrm{N}}$, where $\mu_{\mathrm{BN}}$ is the energy of a $\mathrm{BN}$ pair computed in free standing $h$-BN, and $\mu_{\mathrm{N}}$ is obtained from gas phase $\mathrm{N}_{2} \cdot{ }^{[40]}$

In panel a) of Fig. 4 the different vacancy sites are labelled. We distinguish between sites at different distances with respect to the pore centre. For each species, we compute vacancies at different sites: at the centre of the pore (1), at the border of the pore (2), at the rim ( 3 and 4$)$, or at the wire (5 and 6$)$. The vacancy pairs are combinations of two single ones, and the labels from 1 to 6 also refer to a growing distance from the pore centre. All calculated vacancies on the $h$-BN nanomesh have a lower formation energy than the corresponding defect in free-standing $h$-BN, which are $180 \mathrm{kcal} / \mathrm{mol}$ for B vacancy, $183 \mathrm{kcal} / \mathrm{mol}$ for the $\mathrm{N}$ vacancy, and $197 \mathrm{kcal} / \mathrm{mol}$ for the pair. This is due to the fact that the substrate can partly compensate the dangling bonds that are created upon the removal of boron or nitrogen from $h$-BN. The data points in the top graph of panel b) are the formation energies of the single vacancies. One can observe that the stabilisation is more 
effective when the vacancy is located in the pore or at the rim. Here, the monolayer is relatively close to the $\mathrm{Rh}$ surface and with small deformation of the $h$-BN lattice the broken bonds can be at least partially saturated by the interaction with the metal. Another interesting aspect is that, in most of the cases, the $\mathrm{N}$ vacancies are less stable than the $\mathrm{B}$ vacancies. This is understood to be due to the larger loss in binding energy for $\mathrm{N}$, since the lock-in effect responsible for the formation of the nanomesh is mainly due to the $\mathrm{N}-\mathrm{Rh}$ interaction.

The vacancy pairs are more stable than two single $\mathrm{B}$ and $\mathrm{N}$ vacancies because upon association the number of broken $\mathrm{B}-\mathrm{N}$ bonds is reduced. Also in this case, we notice that the association is particularly stable in the pore and close to the rim, suggesting that once formed, vacancies would remain trapped in the pore. The structure of three of the vacancy pair structures, i.e. $1_{\mathrm{BN}}, 3_{\mathrm{BN}}$ and $6_{\mathrm{BN}}$, are reported in panels c) and d) of Fig. 4. In c) the entire nanomesh unit cell and in d) the local structure around the defect are shown. The $1_{\mathrm{BN}}$ is in the middle of the pore and causes only a local relaxation of the surrounding bond lengths, while the dangling B atoms move slightly towards the substrate. The vacancy pair close to the rim, labelled $3_{\mathrm{BN}}$, turns out to be the most stable. All four dangling atoms interact strongly with one Rh atom that is displaced upward by more than $1 \AA$. The vacancy pair in the wire region causes a relatively large reconstruction, involving more atoms and a significant change in the shape of the rim. An eight-membered ring is formed, including one B-B and one $\mathrm{N}-\mathrm{N}$ bond. Similar larger defects in the wire region have been observed in STM after ion sputtering. ${ }^{[41]}$

\section{Functionalisation}

The covalent chemistry of isolated $h$-BN nanosheets or the nanomesh has become a field of strong interest. Indeed, functionalising $h$-BN is highly attractive in order to make it suitable for use in an expanded range of applications. For example, functionalisation could change its solubility in common solvents or tailor its electronic and magnetic properties. In many reports, non-covalent functionalisation of $h$-BN has been described. ${ }^{[4]}$ In other studies, edge functionalisation has been achieved, even if it usually leads to the destruction of the extended $h$-BN sheets with formation of nanoscale fragments. ${ }^{42]}$ More recently, covalent functionalisation by peroxide-induced hydroxyl groups has been achieved. [43]

We performed DFT simulations to investigate the reaction processes occurring upon chemical functionalisation of $h$-BN sheets, and the structural and electronic properties of $\mathrm{OH}$ - and $\mathrm{H}$-functionalised sheets. We report the results both on the freestanding and the metal-supported monolayers and discuss the differences between them.

We calculate the binding energies upon the functionalisation of a $6 \times 6$ periodic $h$-BN layer with $\mathrm{H}_{2}$, a hydroxyl group or a hydroxyl group together with a hydrogen atom at the nearest-neighbour site. In the initial configuration, the reactants, i.e. $h$-BN layer and the molecular species, are a few $\AA$ apart. The optimal geometry is obtained after the full relaxation of the system. We use $\mathrm{H}_{2}-h \mathrm{BN}$ to designate the $6 \times 6 h$-BN sheet with one $\mathrm{H}$ atom on top of $\mathrm{N}$ and another one on top of the nearest-neighbour B, $\mathrm{OH}-h \mathrm{BN}$ for one hydroxyl group on top of a $\mathrm{B}$ and a hydrogen atom on top of the nearest-neighbour $\mathrm{N}$, and $\mathrm{OH}-h \mathrm{BN}^{*}$ for an $\mathrm{OH}$ functionalising group only. The resulting optimised structures are displayed in Fig. 5.

Some structural parameters in the different optimised geometries are reported in Table 1 . In all cases, we observe the cleavthe local distortion of the planar lattice, as the $\mathrm{B}$ and $\mathrm{N}$ atoms involved in the funcage of the in-plane B-N bond together with

tionalisation are shifted out of plane. As expected, the hybridisation of $\mathrm{N}$ changes from the planar $s p^{2}$ to the tetrahedral $s p^{3}$ configuration when it is protonated.

The relative stability of the different final structures is determined by the binding energies computed from

$$
E_{\text {bind }}=E_{\text {func }}-\left(E_{h \mathrm{BN}}+E_{\text {isol }}\right) \text {, }
$$

where $E_{\text {func }}$ is the energy of the functionalised $h$-BN sheet, $E_{h \mathrm{BN}}$ the energy of the bare $h$-BN sheet and $E_{\text {isol }}$ the sum of the energies of different species that can be involved in the functionalisation. Two examples of how we compute the binding energies are given for the case functionalisation by $\mathrm{H}_{2} \mathrm{O}$ and by $\mathrm{O}_{2}+\mathrm{H}_{2}$

$$
E_{\text {bind }}=E_{\mathrm{OH}-h \mathrm{BN}}-\left(E_{h \mathrm{BN}}+E_{\mathrm{H} 2 \mathrm{O}}\right)
$$
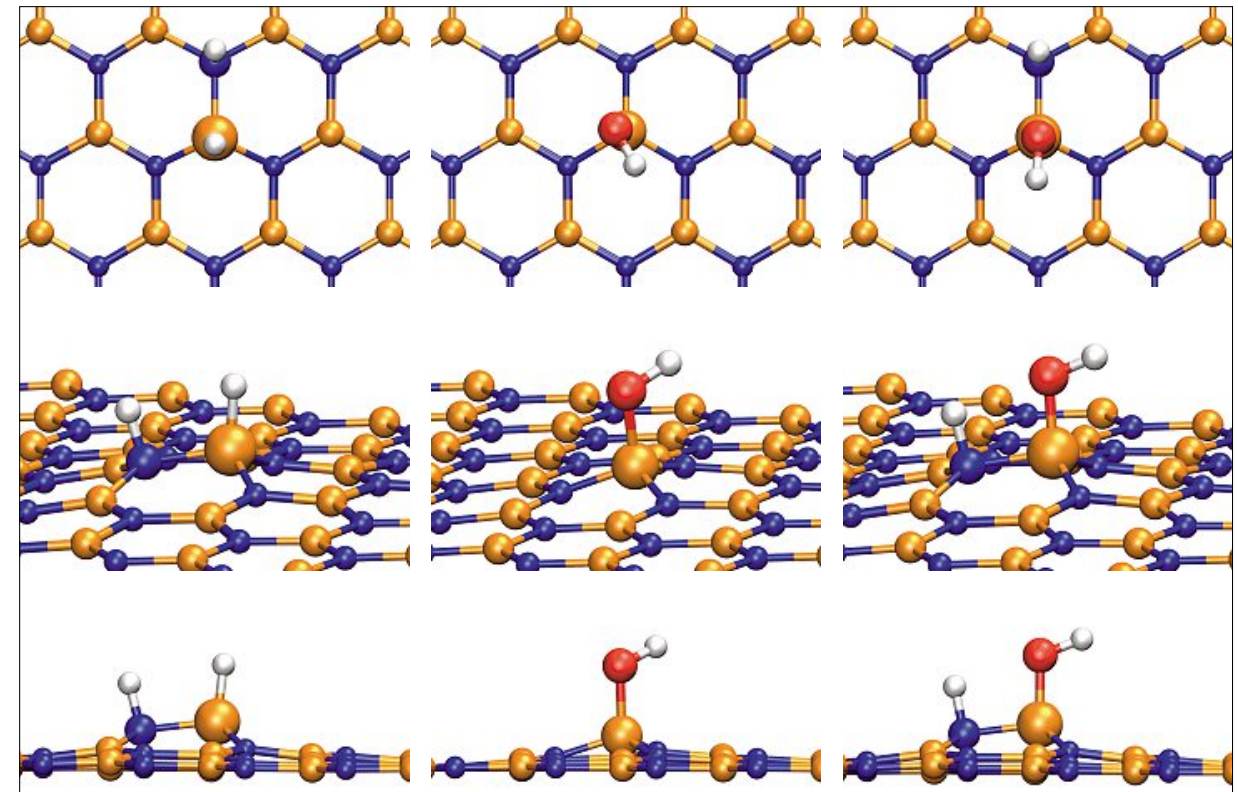

Fig. 5. Optimised geometry after the functionalisation of free-standing $h$-BN with $\mathrm{H}_{2}$ (left), $\mathrm{OH}^{*}$ (centre) and $\mathrm{H}_{2} \mathrm{O}$ (right) ( $\mathrm{O}$ atoms are drawn as red, $\mathrm{H}$ as white, $\mathrm{B}$ as orange, and $\mathrm{N}$ as blue spheres).

Table 1. Structural parameters of functionalised $h-\mathrm{BN}$ sheets. $d(\mathrm{~B}-\mathrm{N})$ is the distance between the $\mathrm{B}$ and $\mathrm{N}$ atoms involved in the functionalisation; $\Delta \mathrm{z}_{\mathrm{B}, \mathrm{N}}$ the vertical shifts of the atoms in the $h$-BN layer involved in the functionalisation; $\theta_{\mathrm{BN}}$ the tetrahedral angle formed by the $\mathrm{B}$ and $\mathrm{N}$ atom with its nearest-neighbour atoms. All distances are given in $\AA$ and angles in degrees.

\begin{tabular}{|l|c|c|c|c|c|}
\hline Structure & $d(\mathrm{~B}-\mathrm{N})$ & $\Delta \mathrm{z}_{\mathrm{B}}$ & $\Delta \mathrm{z}_{\mathrm{N}}$ & $\theta_{\mathrm{B}}$ & $\theta_{\mathrm{N}}$ \\
\hline Bare $h \mathrm{BN}$ & 1.45 & 0 & 0 & 0 & 0 \\
\hline $\mathrm{H}_{2}-h \mathrm{BN}$ & 1.66 & 0.93 & 0.75 & 34 & 18 \\
\hline $\mathrm{OH}-h \mathrm{BN}^{\cdot}$ & 1.53 & 0.62 & 0.16 & 30 & \\
\hline $\mathrm{OH}-h \mathrm{BN}$ & 1.65 & 0.87 & 0.66 & 32 & 17 \\
\hline
\end{tabular}




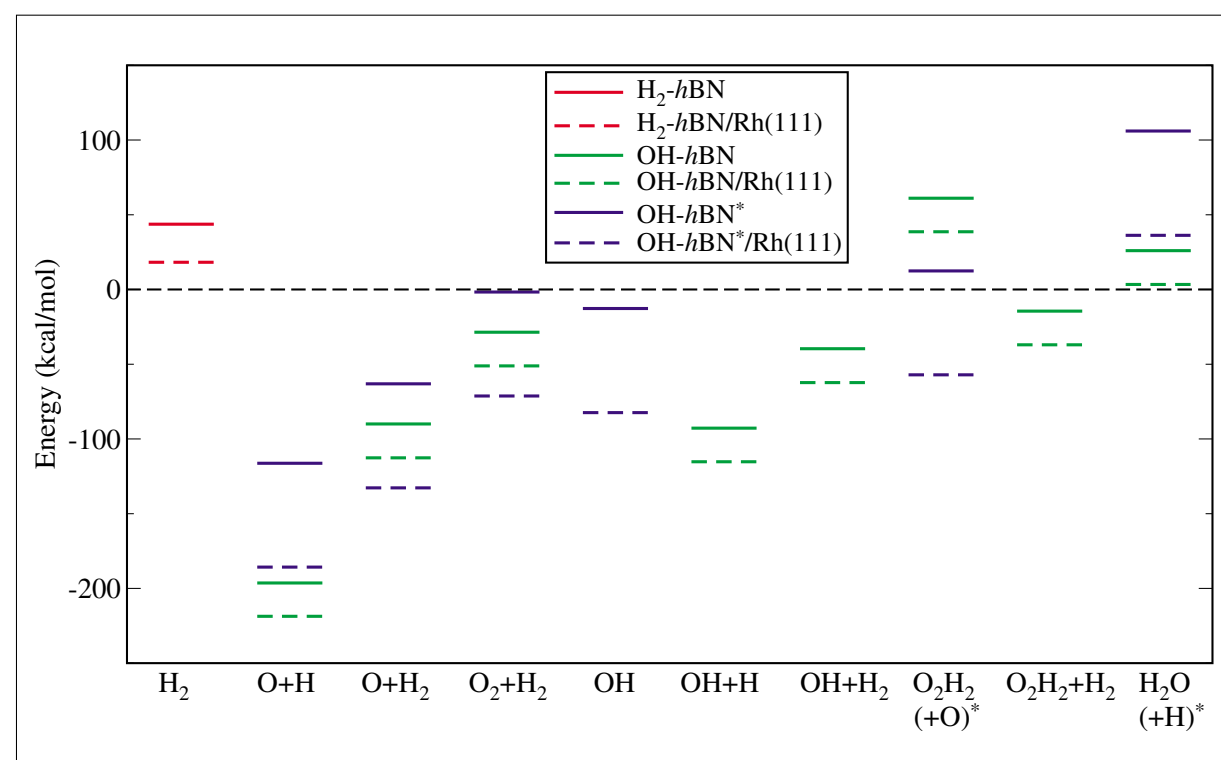

Fig. 6. Binding energies upon three kinds of functionalisation on bare $h$-BN and $h$-BN/Rh(111). For each kind of functionalisation several sets of isolated species (labelled on the horizontal axis) have been considered as the reference and the corresponding binding energy is calculated as described in the text.

Negative values of $E_{\text {bind }}$ indicate a thermodynamically stable functionalisation. The results are gathered in Fig. 6, where the reference isolated species are given on the abscissa. In most of the cases, the functionalisation turns out to be stable. Exceptions are the functionalisations from highly stable species, such as $\mathrm{H}_{2}$ and $\mathrm{H}_{2} \mathrm{O}$. We also notice that the largest binding energies are achieved starting from atomic $\mathrm{O}$ and atomic $\mathrm{H}$ in both $\mathrm{OH}-h \mathrm{BN}$ and $\mathrm{OH}-h \mathrm{BN}^{*}$, and the fully saturated $\mathrm{OH}-h \mathrm{BN}$ structures are in general more stable than the corresponding radical $\mathrm{OH}-h \mathrm{BN}^{*}$ systems.

The same $\mathrm{H}_{2}-h \mathrm{BN}, \mathrm{OH}-h \mathrm{BN}$ and $\mathrm{OH}-h \mathrm{BN}^{\bullet}$ structures have been optimised with $h$-BN supported on $\mathrm{Rh}(111)$ in the (N-top,B-fcc) registry, corresponding to the pore of the nanomesh. The resulting binding energies are reported in Fig. 6 with dashed lines. The interaction with the metal stabilises the functionalisation of $h$-BN by about $25 \mathrm{kcal} / \mathrm{mol}$ for $\mathrm{H}_{2}-h \mathrm{BN}, 33 \mathrm{kcal} /$ $\mathrm{mol}$ for $\mathrm{OH}-h \mathrm{BN}$ and $70 \mathrm{kcal} / \mathrm{mol}$ for $\mathrm{OH}-$ $h \mathrm{BN}^{*}$. The stabilisation is stronger in the $\mathrm{OH}-h \mathrm{BN}{ }^{*}$ structure, because the dangling $\mathrm{N}$ atom can interact with the metal. With reference to $\mathrm{H}_{2}$ and $\mathrm{H}_{2} \mathrm{O}$, the functionalisation is still thermodynamically less stable, but on the metallic substrate the difference is substantially reduced. In particular, the $\mathrm{OH}-h \mathrm{BN}$ structure is only $3 \mathrm{kcal} / \mathrm{mol}$ less stable. We note here that the intact, molecular adsorption is exothermic, by $4 \mathrm{kcal} /$ mol at the free-standing $h$-BN and by 3 $\mathrm{kcal} / \mathrm{mol}$ in the pore of the nanomesh. ${ }^{[21,44]}$

We employ the climbing-image nudged elastic band (CI-NEB) approach ${ }^{[45]}$ to simulate the full process of $\mathrm{H}_{2} \mathrm{O}$ splitting and binding to the substrate, both at the freestanding monolayer and supported $h$-BN.
These simulations yield the minimum energy path (MEP) of the reaction and an evaluation of the activation barrier on the potential energy surface. The initial state (IS) is the water molecule $2.8 \AA$ above $h$-BN, with the $\mathrm{O}$ atom on top of a B atom, one $\mathrm{O}-\mathrm{H}$ bond along the $\mathrm{B}-\mathrm{N}$ bond, and the other one pointing outwards. The final state (FS) is the previously optimised $\mathrm{OH}-$ $h \mathrm{BN}$ structure. The complete band is described by 24 replicas, initially obtained by linear interpolation between IS and FS, and then optimised with the NEB procedure. The resulting MEPs are displayed in Fig. 7. Both reactions, on bare and supported

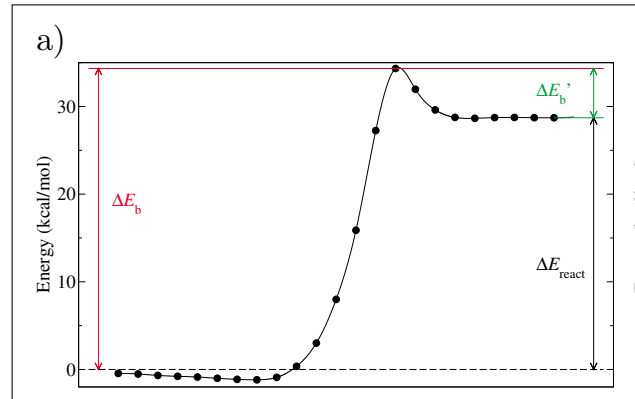

c)

d)
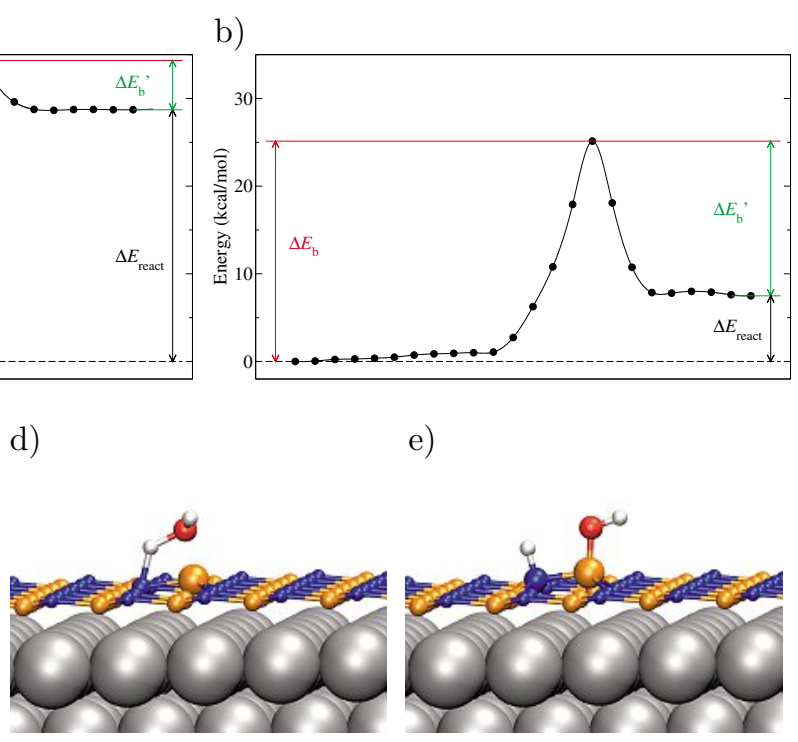

e)
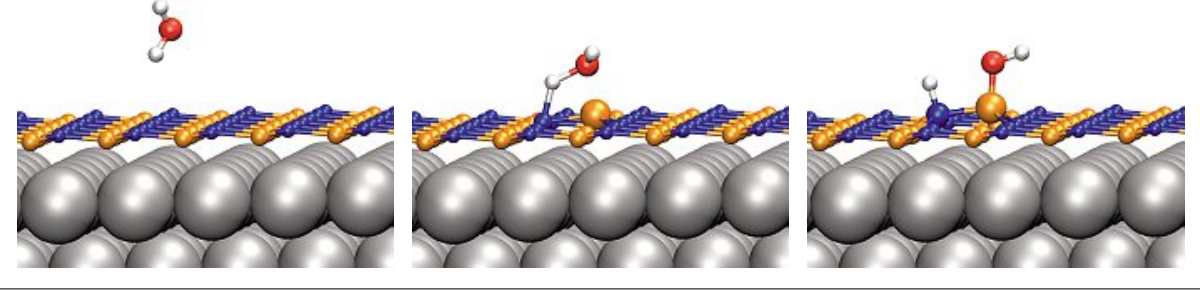

Fig. 7. The potential energy profile along MEP upon the covalent functionalisation of a) free-standing $h$-BN and b) the $h$-BN/Rh(111) nanomesh with a water molecule. c) Initial, d) transition, and e) final state configuration in the latter case.
$h$-BN, are endothermic and activated, with the corresponding activation barriers $\Delta E_{\mathrm{b}}$ of 34 and $25 \mathrm{kcal} / \mathrm{mol}$. The transition state (TS) configuration at the maximum of the profle, together with the IS and FS shown in the d), c) and e) panels of Fig. 7 respectively. IS, TS and FS configurations on the freestanding $h$-BN are very similar. At the TS the $\mathrm{O}-\mathrm{H}$ bond is slightly elongated, but the $\mathrm{O}$ atom and $\mathrm{H}$ atoms are already close to the $\mathrm{B}$ and $\mathrm{N}$ binding sites, respectively. The endothermicity in the reaction energy, $\Delta E_{\text {react, }}$ is much lower on the supported $h$ - $\mathrm{BN}$, thus making the dissociation reaction, and alongside the functionalisation, more accessible there. The energetics here thus follows qualitatively the empirical Bronsted-Evans-Polanyi (BEP) principle about the height of the activation barriers scaling (linearly) with the corresponding reaction energies. The probability of observing functionalisation with water at the free-standing $h$-BN is further reduced due to the low barrier, $\Delta E_{\mathrm{b}}$ ', for back reaction of water re-association and subsequent desorption.

\section{Summary}

DFT-based calculations have been shown to yield a reliable description of single layer $h$-BN on metal surfaces. Using the same established simulation protocol, we have investigated different types of chemical reactions involving the $h$-BN/ $\mathrm{Rh}(111)$ system, either as a template or directly as a reactant.

In agreement with experiment, we find that the highly symmetric molecule CHP 
adsorbs preferentially in the pore of the nanomesh. Further calculations on single and multiple dehalogenation of CHP reveal a strong interaction of radical-type carbon atoms of CHP with boron atoms of the underlying $\mathrm{BN}$ sheet. The anchoring provided by this interaction together with the hexagonal pattern of the $h$-BN makes further dehalogenation more favourable at the meta positions. This mechanism gives a convincing explanation of the fact that STM tip-induced dehalogenation is limited to $5,5^{\prime \prime}, 5^{\prime \prime \prime}$ '-I3-CHP and that the same molecule is the most abundant species found after thermal treatment of the samples.

The formation of boron or nitrogen vacancies can be seen as a first step before subsequent functionalisation or as a functionalisation by itself. Vacancies can be introduced by exposure of $h$-BN to low energy ions and can be annealed at elevated temperatures due to their mobility. STM experiments have revealed an accumulation of defects at the rim and the wire of the nanomesh. By calculating the formation energies of different defect types, we are able to explain the experimental distribution of defects. In particular, we find that $\mathrm{N}$ vacancies are less stable than $B$ vacancies and that the strong interaction of the pore regions allows for an increased stabilisation of vacancies in the pore. This also suggests that vacancies generated in the pore will not leave during annealing. It is further found that $\mathrm{BN}$ vacancy pairs are more stable than the corresponding single vacancies and that they have a tendency to accumulate towards the rim.

Finally, functionalisation of $h$-BN with hydroxyl and hydrogen is explored as a test case for covalent functionalisation. We find that the most stable products are formed by reaction of $\mathrm{OH}$ with boron in the pore region of the nanomesh. Using NEB calculations, we can further show that the transition state energy is considerably reduced in the pore. This leads us to the conclusion that one can expect a strong templating effect for radical-based functionalisation of the $h$-BN/Rh(111) nanomesh.

The calculations presented here are further evidence for the predictive power of modern electronic structure simulations and the insight that can be won if such simulations are used together with experiments on complex chemical structures.

\section{Received: May 14, 2014}

[1] For reviews see for example refs [2-4].

[2] C. Oshima, A. Nagashima, J. Phys: Condens. Matt. 1997, 9, 1.

[3] H. Ma, M. Thomann, J. Schmidlin, S. Roth, M. Morscher, T. Greber, Front Phys. China 2010, $5,387$.

[4] Y. Lin, J. W. Connell, Nanoscale 2012, 4, 6908.

[5] M. Corso, W. Auwärter, M. Muntwiler, A. Tamai, T. Greber, J. Osterwalder. Science 2004, 303, 217.

[6] A. B. Preobrajenski, A. S. Vinogradov, Nils Mårtensson, Surf. Sci. 2005, 582, 21.

[7] S. Joshi, D. Ecija, R. Koitz, M. Iannuzzi, A. P. Seitsonen, J. Hutter, H. Sachdev, S. Vijayaraghavan, F. Bischoff, K. Seufert, J. Barth, W. Auwärter, NanoLett. 2012, 12, 5821.

[8] T. Brugger, S. Günnther, B. Wang, J. H.Dil, M.-L. Bocquet, J. Osterwalder, J. Wintterlin, T. Greber. Phys. Rev. B 2009, 79, 045407.

[9] a) S. Berner, M. Corso, R. Widmer, O. Gröning, R. Laskowski, P. Blaha, K. Schwarz, A. Goriachko, H. Over, S. Gsell, M. Schreck, H. Sachdev, T. Greber, J. Osterwalder. Angew. Chem. Int. Ed. 2007, 46, 5115; b) http://www. Nanomesh.ch/

[10] M. Morscher, M. Corso, T. Greber, J. Osterwalder, Surf. Sci. 2006, 600, 3280

[11] F. Müller, S. Hüfner, H. Sachdev, R. Laskowski, P. Blaha, K. Schwarz, Phys. Rev. B 2010, 82 , 113406.

[12] A. B. Preobrajenski, A. S. Vinogradov, M. L. Ng, E. Ćavar, R. Westerstrom, A. Mikkelsen, E. Lundgren, N. Mårtensson, Phys. Rev. B 2007, $75,245412$.

[13] J. Wintterlin, M.-L. Bocquet, Surf. Sci. 2009 603,1841

[14] H. Dil, J. Lobo-Checa, R. Laskowski, P. Blaha, S. Berner, J. Osterwalder, T. Greber, Science 2008, 319, 1824

[15] R. Koitz, A. P. Seitsonen, M. Iannuzzi, J. Hutter, Nanoscale 2013, 5, 5589.

[16] M. Iannuzzi, F. Tran, R. Widmer, T. Dienel, K. Radican, Y. Ding, J. Hutter, O. Gröning, Phys. Chem. Chem. Phys. 2014, 16, 12374.

[17] T. Brugger, H. Ma, M. Iannuzzi, S. Berner, A. Winkler, J. Hutter, J. Osterwalder, T. Greber, Angew. Chem. Int. Ed. 2010, 49, 6120.

[18] S. Joshi, F. Bischoff, R. Koitz, D. Ecija, K. Seufert, A. P. Seitsonen, J. Hutter, K. Diller, J. I. Urgel, H. Sachdev, J. V. Barth, W. Auwärter, ACS Nano 2014, 8, 430.

[19] H. Ma, T. Brugger, S. Berner, Y. Ding, M. Iannuzzi, J. Hutter, J. Osterwalder, T. Greber, ChemPhysChem 2010, 11, 399.

[20] H. Ma, Y. Ding, M. Iannuzzi, T. Brugger, S. Berner, J. Hutter, J. Osterwalder, T. Greber, Langmuir 2012, 28, 15246.
[21] Y. Ding, M. Iannuzzi, J. Hutter, J. Phys. Chem. C 2011, 115, 13685.

[22] Y. Ding, M. Iannuzzi, J. Hutter, J. Phys.: Condens. Matt. 2012, 24, 445002

[23] CP2k developers' group under the terms of the GNU General Public Licence; see http://www. CP2K.org/, 2014

[24] J. Hutter, M. Iannuzzi, F. Schiffmann, J. VandeVondele, Wiley Interdisc. Rev-Comput. Mol. Sci. 2014, 4, 15

[25] S. Goedecker, M. Teter, J. Hutter, Phys. Rev. B 1996, 54, 1703.

[26] C. Hartwigsen, S. Goedecker, J. Hutter, Phys. Rev. B 1998, 58,3641 .

[27] M. Krack, Theor. Chem. Acc. 2005, 114, 145.

[28] J. P. Perdew, K. Burke, M. Ernzerhof, Phys. Rev. Lett. 1996, 77, 3865.

[29] J. P. Perdew, K. Burke, M. Ernzerhof, Phys. Rev. Lett. 1997, 78, 1396.

[30] Y. Zhang, W. Yang, Phys. Rev. Lett. 1998, 80, 890.

[31] S. Grimme, J. Antony, S. Ehrlich, H. Krieg, J. Chem. Phys. 2010, 132, 154104.

[32] J. VandeVondele, M. Krack, F. Mohamed, M. Parrinello, T. Chassaing, J. Hutter, Comput. Phys. Commun. 2005, 167, 103 .

[33] P. Bendt, A. Zunger, Phys. Rev. B 1982, 26, 3114.

[34] J. Gómez Díaz, Y. Ding, R. Koitz, A. P. Seitsonen, M. Iannuzzi, Jürg Hutter, Theor. Chem. Acc. 2013, 132, 1350.

[35] J. Cai, P. Ruffieux, R. Jaafar, M. Bieri, T. Braun, S. Blankenburg, M. Muoth, A. P. Seitsonen, M. Saleh, X. Feng, K. Müllen, R. Fasel, Nature 2010, 466, 470.

[36] M. Bieri, M.-T. Nguyen, O. Gröning, J. Cai, M. Treier, K. Aitt-Mansour, P. Ruffieux, C. A. Pignedoli, D. Passerone, M. Kastler, K. Müllen, R. Fasel, J. Am. Chem. Soc. 2010, 132, 16669.

[37] T. Dienel, J. Gómez Díaz, A. P. Seitsonen, R. Widmer, M. Iannuzzi, K. Radican, H. Sachdev, K. Müllen, J. Hutter, O. Gröning, ACS Nano 2014, 8,6571 .

[38] H. Cun, M. Iannuzzi, A. Hemmi, S. Roth, J. Osterwalder, T. Greber, Nano. Lett. 2013, 13, 2098.

[39] L. H. De Lima, H. Cun, A. Hemmi, T. Kälin, T. Greber, Rev. Sci. Instrum. 2013, 84, 126104.

[40] S. Okada, Phys. Rev. B 2009, 80, 161404.

[41] H. Cun, M. Iannuzzi, A. Hemmi, J. Osterwalder, T. Greber, ACS Nano 2014, 8, 7423 .

[42] Y. Lin, T. V. Williams, T.-B. Xu, W. Cao, H. E. Elsayed-Ali, J. W. Connell, J. Phys. Chem. C 2011, 115, 2679.

[43] T. Sainsbury, A. Satti, P. May, Z. Wang, I. McGovern, Y. K. Gun'ko, J. Coleman, J. Am. Chem. Soc. 2012, 134, 18758

[44] Y. Ding, 'Investigation of Molecule Adsorption on h-BN Nanomesh: Water and Phthalocyanine', $\mathrm{PhD}$ thesis, University of Zurich, 2012.

[45] G. Henkelman, B. P. Uberuaga, H. Jónsson, $J$. Chem. Phys. 2000, 113, 9901. 